This item was submitted to Loughborough's Research Repository by the author.

Items in Figshare are protected by copyright, with all rights reserved, unless otherwise indicated.

\title{
Mixtures of catanionic surfactants can be superspreaders: Comparison with trisiloxane superspreader
}

PLEASE CITE THE PUBLISHED VERSION

http://dx.doi.org/10.1016/j.jcis.2015.08.024

PUBLISHER

(C) Elsevier

VERSION

AM (Accepted Manuscript)

\section{PUBLISHER STATEMENT}

This work is made available according to the conditions of the Creative Commons Attribution-NonCommercialNoDerivatives 4.0 International (CC BY-NC-ND 4.0) licence. Full details of this licence are available at: https://creativecommons.org/licenses/by-nc-nd/4.0/

\section{LICENCE}

CC BY-NC-ND 4.0

\section{REPOSITORY RECORD}

Kovalchuk, Nina, Alix Barton, Anna Trybala, and Victor Starov. 2017. "Mixtures of Catanionic Surfactants Can Be Superspreaders: Comparison with Trisiloxane Superspreader”. figshare. https://hdl.handle.net/2134/24222. 


\section{Elsevier Editorial System(tm) for Journal of Colloid and Interface Science}

Manuscript Draft

Manuscript Number:

Title: Mixtures of catanionic surfactants can be superspreaders: comparison with trisiloxane superspreader.

Article Type: Full length article

Section/Category: D. Interfacial Processes, Capillarity and Wetting

Keywords: sodium alcane sulfonates, interfacial tension, spreading kinetics, spreading factor, polyethylene substrate

Corresponding Author: Prof. Victor Starov, MSc, PhD, DSc

Corresponding Author's Institution: Loughborough University

First Author: Victor Starov, MSc, PhD, DSc

Order of Authors: Victor Starov, MSc, PhD, DSc; Nina Kovalchuk, Dr; Anna Trybala, Dr; Alix Barton

Abstract: Kinetics of spreading of mixed solutions of cationic and anionic surfactants over highly hydrophobic substrate such as polyethylene is investigated. It is shown that due to synergetic effect these solutions can wet hydrophobic substrates nearly as effective as solutions of trisiloxane superspreader BT-278. The spreading factor reaches $70 \%$ of that of superspreader for the most effective mixed solution. At room humidity ( $40 \%$ ) spread area has a maximum vs concentration. However, the maximum was not observed at higher humidity $80 \%$. The spreading rate of mixed solutions is smaller than that of superspreader despite the same spreading exponent $0=0.5$.

Suggested Reviewers: Stanislav Dukhin Dr Professor , New Jersey Institute of Technology sdukhin@bestweb.net

World leading expert in the aera

Vladimir Sobolev Dr Professor, Moscow Institute of Physical Chemistry vladimir.sobolev@mail.ru World leading exert in the area

David Brutin Dr

Associate Professor, University of Marseulle david.brutin@univ-amu.fr

World leading exert in the area

Manuel Velarde Dr Professor, Instituto Pluridisciplinar mvelarde@pluri.ucm.es World leading expert in the area 


\author{
Hans Yurgen Butt Dr \\ Professor, Max Plan Institute \\ butt@mpip-mainz.mpg.de \\ World leading expert in the area \\ Tatiana Gambaryan Dr \\ Professor, University of Darmstadt \\ gtatiana@ttd.tu-darmstadt.de \\ World leading expert in the area \\ Emil Chibowsky Dr \\ Professor, University of Lublin \\ emil.chibowski@umcs.lublin.pl \\ world leading expert in the area \\ John Gaydos Dr \\ Associate Professor, Carleton University \\ jgaydos@mae.carleton.ca \\ world leading expert in the area
}




\section{Graphical abstract}

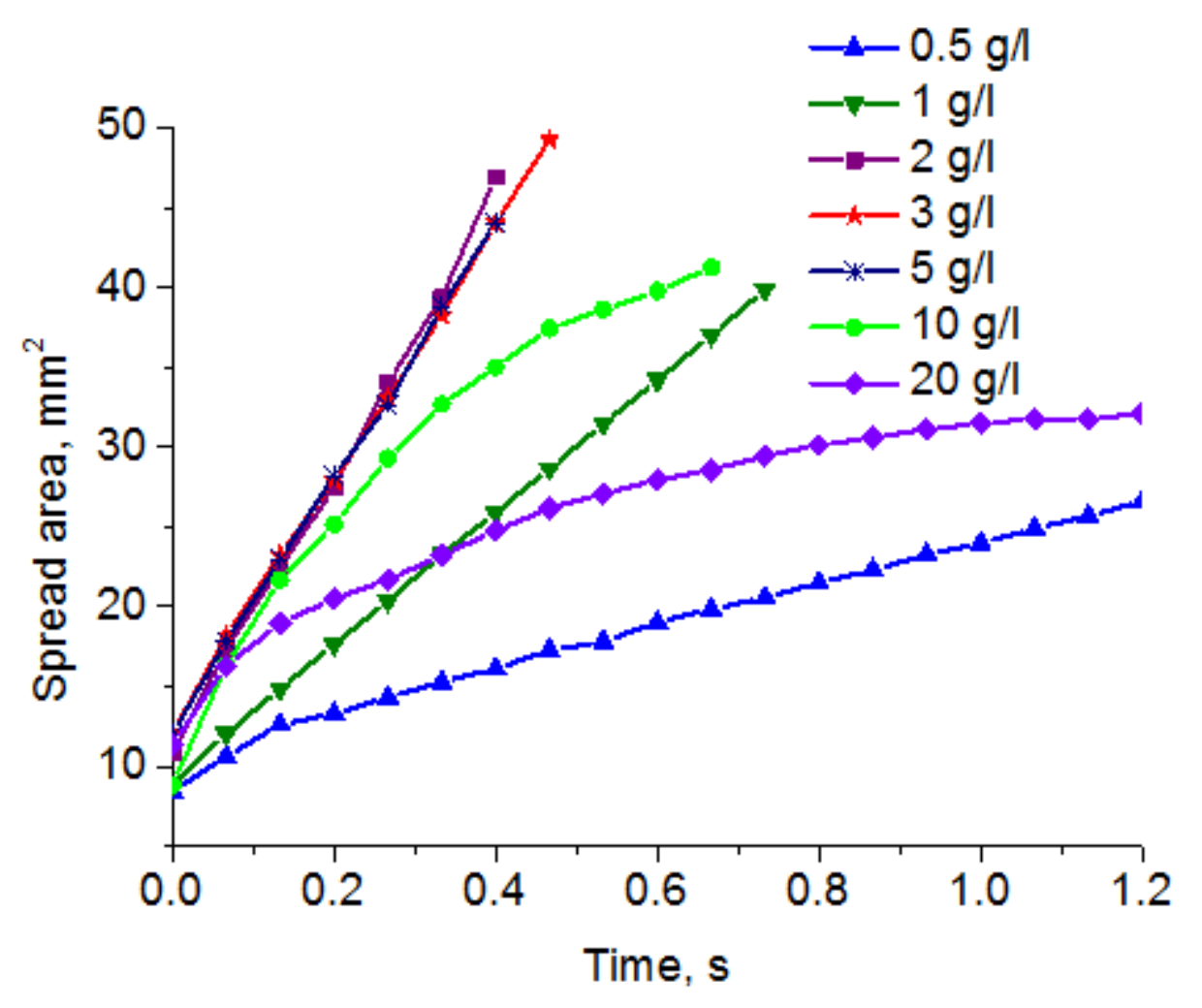

\section{Highlights:}

- spreading of mixed solutions of cationic and anionic surfactants

- synergetic effect of these solutions

- wetting of hydrophobic substrates nearly as superspreader

- spreading rate of mixed solutions is smaller than that of superspreader

"Corresponding Author: Victor Starov, Department of Chemical Engineering, Loughborough University, Loughborough, LE113TU, UK. E-mail: v.m.starov@ @ lboro.ac.uk, Phone: +44(0)1509 222508, Fax: $+44(0) 1509223923$ 
1

2

\title{
Mixtures of catanionic surfactants can be superspreaders: comparison with trisiloxane superspreader.
}

\author{
N.M. Kovalchuk ${ }^{1,2}$, A. Barton ${ }^{1}$, A. Trybala ${ }^{1}$, V. Starov ${ }^{*}$ \\ ${ }^{1}$ Department of Chemical Engineering, Loughborough University, \\ Loughborough, LE 11 3TU, UK \\ ${ }^{2}$ Institute of Biocolloid Chemistry, Kiev, 03142, Ukraine
}

\begin{abstract}
Kinetics of spreading of mixed solutions of cationic and anionic surfactants over highly hydrophobic substrate such as polyethylene is investigated. It is shown that due to synergetic effect these solutions can wet hydrophobic substrates nearly as effective as solutions of trisiloxane superspreader BT-278. The spreading factor reaches $70 \%$ of that of superspreader for the most effective mixed solution. At room humidity (40\%) spread area has a maximum vs concentration. However, the maximum was not observed at higher humidity $80 \%$. The spreading rate of mixed solutions is smaller than that of superspreader despite the same spreading exponent $\alpha=0.5$.
\end{abstract}

Keywords: sodium alcane sulfonates, interfacial tension, spreading kinetics, spreading factor, polyethylene substrate.

${ }^{*}$ Corresponding author: V.M.Starov@lboro.ac.uk 


\section{Introduction}

Improvement of wettability of hydrophobic substrates by aqueous formulations is of great importance for various industries such as oil recovery; printing, painting and coating; treatment of plants and soils with pesticides, herbicides and fertilisers in agriculture; eye lubricants and treatment of respiratory dysfunctions in medicine etc. Water does not wet low energy surfaces because it has a very high surface energy (surface tension $\sim 72 \mathrm{mN} / \mathrm{m}$ at room temperature) as compared with other liquids except for liquid metals. Surfactants lower both liquid/vapour and solid/liquid interfacial tensions and in this way facilitate wetting. Trisiloxane surfactants are the most effective known wetting agents frequently referred to as superspreaders. Trisiloxanes promote complete wetting by aqueous solutions of hydrophobic substrates, on which pure water has contact angle around $100{ }^{\circ}$ and higher (polyethylene, polypropylene, parafilm) [1, 2]. Aqueous trisiloxane solution can cover area up to 100 times larger than that covered by pure water and spreading process occurs relatively fast on the time scale of tens of seconds.

The most famous trisiloxane superspreader has a structure presented in Fig. 1 and is known under trade names Silwet-L77 and BREAK-THRU S 278; some other abbreviations, including those related to the structure, are also used in literature (see $[1,2]$ for examples).

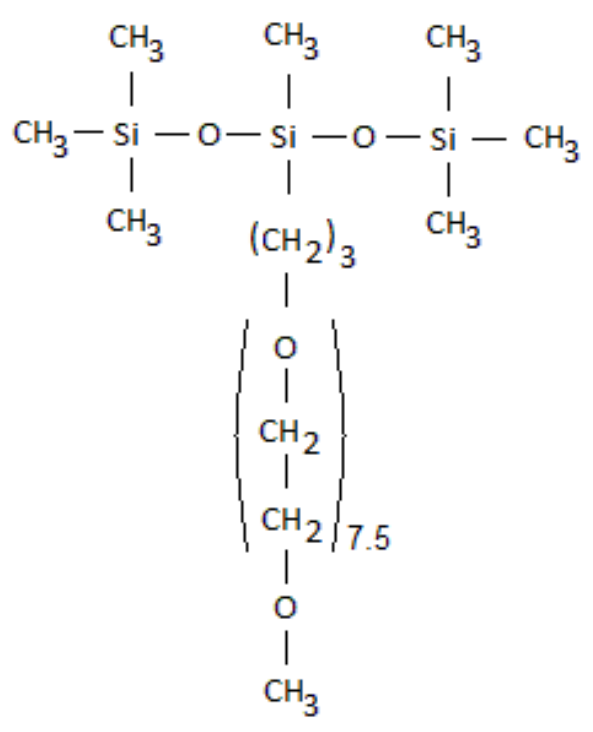

Fig. 1.The structure of trisiloxane surfactant known under the trade names SilwetL77 and BREAK-THRU S 278. 
Ability of trisiloxane surfactants to wet completely hydrophobic substrates is related to their very low surface tension, 20-22 $\mathrm{mN} / \mathrm{m}$ [3-5] and good adsorption on hydrocarbon surfaces: according to [3] interfacial tension between aqueous trisiloxane solutions and tetradecane is close to zero.

However, the very high rate of spreading of trisiloxane solutions is much less understood. In the case of pure liquids complete spreading is driven by interplay of capillary forces, disjoining pressure and viscous resistance in the vicinity of the three-phase contact line [6]. The latter results in well-known Tanner law [6, 7], with $\alpha=0.1$ :

$R(t)=A t^{\alpha}$

or

$S(t)=A_{1} t^{2 \alpha}$

where $A=K\left(\frac{V^{3} \sigma_{l v}}{\mu}\right)^{\alpha}, \quad \mathrm{A}_{1}=\mathrm{A}^{2}, \mathrm{R}(\mathrm{t})$ is the radius of base of a spreading droplet, $\mathrm{S}(\mathrm{t})$ is its area, $K$ and $K_{1}$ are the numerical (dimensionless) coefficients depending (rather weakly [6]) on parameters of disjoining pressure isotherm (Hamaker constant), $V$ is the droplet volume, $\sigma_{l v}$ is the liquid/vapour surface tension, $\mu$ is the dynamic viscosity of the liquid and $t$ is time.

Assuming the same mechanism of spreading, one can expect that surfactant solutions should spread with the same rate or even slower than pure liquids in the case of complete wetting, because at spreading of surfactant solutions two extra relaxation processes are added: adsorption of surfactant molecules at liquid/vapour and solid/liquid interfaces. It was proven in [8] that a very slow spreading kinetics of solutions of fluoro-surfactant Novec FC-4430 is directly related to its slow adsorption kinetics.

However, comparison of spreading kinetics of solutions of trisiloxane surfactants and pure liquids show a substantial difference in favour of the surfactant solutions (Fig. 2), with $\alpha=0.1$ for silicone oil and $\alpha=0.5$ for aqueous solution of trisiloxane surfactant. 
Spreading kinetics $R(t) \sim t^{0.5}$ is a characteristic feature of diffusion governed processes. Exactly this power law kinetics was found for spreading of pure trisiloxane surfactant (without water added) [9], however, the spreading was very slow, with spreading rate in the range from $\mathrm{mm}^{2} /$ day to $\mathrm{mm}^{2} /$ hour depending on humidity. An increase in humidity from 30 to $100 \%$ resulted in the 10 times increase of apparent diffusion coefficient. Therefore interaction with water is essential for understanding the fast spreading mechanism.

It is well known [10] that the main energy in the curse of spreading is burned in a vicinity of three phase contact line (inside thin precursor film in front of the apparent three phase contact line). The fast spreading of superspreders suggests that it proceeds according to a different mechanism: cannot be thin precursor films in front of the moving apparent three phase contact line in the case of spreading over hydrophobic substrates. It was the reason why a caterpillar motion in the vicinity of the three phase contact line has been suggested as an explanation of the 
superspreading phenomenon [11]. The caterpillar motion in the case of advancing three phase contact line has been suggested earlier in [12] based on the s-shape of disjoinining pressure isotherm of aqueous solutions. The disjoining pressure isotherm of trisiloxane solutions is still unknown, that is, the caterpillar motion in the case of superspreading is to be confirmed.

However, there is a possibility that Marangoni flow [13] is also an essential contribution to the superspreading phenomenon.

To elucidate the precise mechanism and predict spreading performance based on the known surfactant properties, additional investigations are required, in particularly those comparing spreading of various surfactants.

It should be emphasised that the spreading performance depends essentially on the experimental conditions such as ambient humidity, substrate roughness and even the protocol of the solution preparation [14, 15]. For example, according to [15] sonicated solution of trisiloxane surfactant spread about 2.5 times faster than the hand shaken one. That is why it is extremely important to perform comparative studies using identical substrates, identical ambient conditions and identical experimental protocols. Results of such comparative study are presented below.

It is well known that solutions of other (non-trisiloxane) surfactants, both non-ionic (oxiethylated alcohols) [16] and ionic (sodium bis(2-ethylhexyl) sulfosuccinate, AOT, and didodecyldimethylammonium bromide) [17] demonstrate a fast spreading with $S \sim t$, but on partially wetted substrates characterised by the contact angle of pure water $\sim 50-70^{\circ}$. It is suggested below to use the synergism in the performance of surfactant mixtures [18] to get the superspreading on highly hydrophobic substrates such as polyethylene without trisiloxane superspreaders. Promising candidates for this are mixtures of cationic and anionic surfactants, catanionic mixtures [19, 20]. Below the wetting performance of series of catanionic mixtures related to their surface propertiesis analysed, and compared with solutions of trisiloxane superspreader (Fig. 1). 


\section{Experimental}

Dodecyltrimethylammonium bromide, DoTAB, (Fluka, 99\%); sodium 1decanesulfonate, SDeS, (Fluka, 99\%);sodium 1-octanesulfonate monohydrate, SOcS, (Fluka, 99\%);sodium 1-heptanesulfonate monohydrate, SHepS, (Fluka, 99\%); sodium 1-hexanesulfonate monohydrate, SHexS, (Fluka, 99\%); BREAK-THRU S 278, BT-278, (gift from Evonik); silicone oil, Brookfield viscosity standard; heptane (HCROMASOLV®, Sigma-Aldrich, 99\%); iso-propanol (Fisher Scientific, analytical reagent grade) have been used without any additional purification. All solutions have been prepared in ultra-pure water produced by Millipore $Q(15 \mathrm{M} \Omega \mathrm{cm})$.

Low density polyethylene film, PE (GoodFellow), thickness $0.05 \mathrm{~mm}$, has been cut into pieces $4 \times 4 \mathrm{~cm}$, washed for $15 \mathrm{~min}$ with iso-propanol in ultrasonic bath, rinsed with plenty of water and dried in an oven at $50{ }^{\circ} \mathrm{C}$. The film roughness measured by AFM is $R_{r m s}=34.5 \pm 1.2 \mathrm{~nm}$ at scan size $80 \mu \mathrm{m}$ [20]. The film was placed on the microscopic glass slide support $\left(R_{r m s}=1.9 \pm 0.2 \mathrm{~nm}\right)$. Contact angle of water on this combined substrate was $101 \pm 3^{\circ}$.

Contact angle and interfacial tension have been measured with DSA-100 (Kruss) using bubble/drop shape analysis. It is impossible to measure directly liquid/solid interfacial tension. It was the reason why water/heptane interface was used as a representative model. To prevent depletion of aqueous solutions due to partition into the oil phase the corresponding interfacial tension was measured using heptane droplet placed into aqueous solution with v:v ratio about 1000. Consequent measurements on the series of droplets (up to 10) have shown the same value of equilibrium interfacial tension proving that depletion due to partition can be neglected.

All spreading experiments have been performed at room temperature $T=23 \pm 1^{\mathrm{C}} \mathrm{C}$ and relative humidity $\mathrm{RH}=40 \pm 5$ if not otherwise stated. Kinetics of spreading was measured using the series of the images of spreading droplets (top view) taken by video camera at $15 \mathrm{fps}$. The areas have been calculated using ImageJ free software. The spreading factor was calculated as a ratio of maximum spread area and the area covered by a droplet of pure water of the same volume, $14 \mu \mathrm{l}$. The long-time kinetics was measured using $14 \mu \mathrm{l}$ droplets, whereas the short-time kinetics was measured using $2 \mu$ droplets. 


\section{Results and discussion}

Equilibrium contact angle of a liquid droplet on a solid substrate is determined by the energy balance given by the Young equation:

$\cos \theta=\frac{\sigma_{s v}-\sigma_{s l}}{\sigma_{l v}}$

where $\sigma_{s v}, \sigma_{s l}$, and $\sigma_{l v}$ are the solid/vapour, solid/liquid and liquid/vapour interfacial tensions. As experimentally measurable quantities are advancing and receding contact angles, the best approximation for $\theta$ is considered to be static advancing contact angle [22]. Complete wetting occurs when the right hand site of Eq. (2) becomes positive, i.e.:

$\sigma_{s v}-\left(\sigma_{s l}+\sigma_{l v}\right)>0$

and contact angle does not exist anymore $(\cos \theta>1)$. Inequality (3) means that it is energetically favourable to replace the solid/vapour interface by two other interfaces: solid/liquid and liquid/vapour. Eq. (3) shows that a decrease of solid/liquid and liquid/vapour surface tension favours wettability of the solid surface.

Minimum attainable interfacial tension at water/air and water/alcane interfaces due to adsorption of individual ionic surfactants used is essentially higher than that of trisiloxane solutions: DoTAB enables lowering the water/air surface tension to $\sim 38$ $\mathrm{mN} / \mathrm{m}$ and water/alcane interfacial tension to $\sim 5 \mathrm{mN} / \mathrm{m}$ [22]. Very close value of water/air surface tension was found below for SOcS, whereas water/heptane interfacial tension was even higher than that of DoTAB: 10 mN/m (Fig. 3). 
Fig. 3. Interfacial tension isotherms of SOcS: 1 - at water/air interface, 2 - at water/heptane interface. For this surfactant $\mathrm{cmc} \sim 100 \mathrm{~g} / \mathrm{l}$ and it does not wet completely polyethylene $\left(\mathrm{CA} \sim 66^{\circ}\right)$ (see below).

Comparison of values of minimum attainable interfacial tensions (Fig. 3) with the data for the trisiloxane superspreaders $(20-22 \mathrm{mN} / \mathrm{m}$ at water/air [3-5] and close to zero at water/alcane [3] interface) shows that they are considerably higher than corresponding values found for trisiloxanes. The comparison shows that solutions of individual ionic surfactants under investigation should spread worse than those of trisiloxane surfactants. Indeed individual ionic surfactants used demonstrated only partial wetting on the polyethylene substrate with contact angle in the range of $66.5 \pm 1^{\circ}$ for solutions of SOcS at concentrations above cmc $(\sim 100 \mathrm{~g} / \mathrm{l})$.

When cationic and anionic surfactants are mixed together then electrostatic repulsion between individual ionic surfactant molecules is replaced by electrostatic attraction between oppositely charged parts of molecules. This results in closer packing of molecules in adsorption layers and, consequently, lower interfacial tension on both water/air and water/alcane interface as can be seen in Figs. 4 and 5 . 


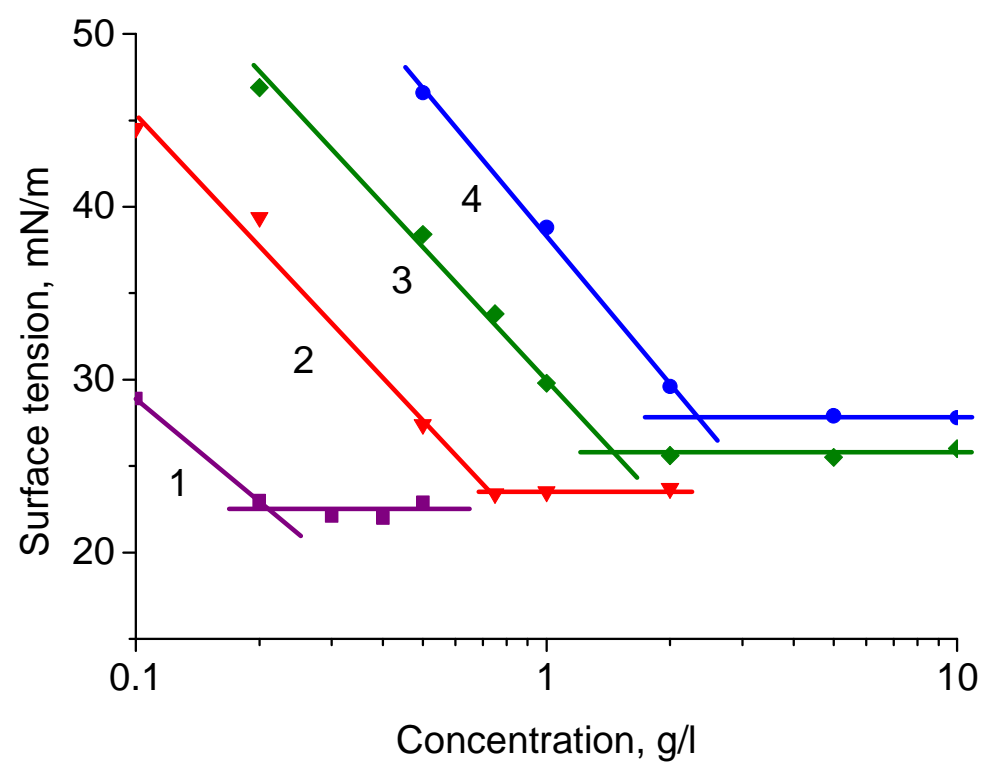

Fig. 4. Equilibrium interfacial tension at water/air interface for $v: v=1: 1$ mixture of DoTAB with: 1 - SDeS, 2 - SOcS, 3 - SHepS, 4 - ShexS.

Cmc can be found from Fig. 4 as the intersection points of two straight lines. It should be noted that for trisiloxane surfactants superspreading begins at concentration called critical wetting concentration (cwc) which is several times higher than their critical aggregation concentration (cac). Cwc for catanionic mixtures can be found from Fig. 6, but for these mixtures difference between $\mathrm{cmc}$ and $\mathrm{cwc}$ is inside the experimental error, i.e. there is no essential difference. At concentrations above $\mathrm{cmc}$ the water/heptane interfacial tension was very low: it was impossible to measure interfacial tensions below $1.5 \mathrm{mN} / \mathrm{m}$ using drop shape analysis, because spontaneous emulsification occurred preventing the drop formation. 
Water/air interfacial tension increased with the decrease of chain length of sodium alcane sulfonate. The low values of water/air interfacial tension, which are comparable with that for BT-278, enabled complete wetting for all mixtures except SHepS. For mixture DoTAB with SHexS only partial wetting occurred with contact angle about $13^{\circ}$ at concentrations above cmc.

In catanionic mixtures both micellisation and the macroscopic phase separation can take place. For the mixture SDeS+DoTAB the latter resulted in crystallization with the limit of solubility being lower than cmc (see [20] for details and it effect on the spreading performance). For the mixture SOcS+DoTAB the liquid phase separation becomes clearly visible at concentration $5 \mathrm{~g} / \mathrm{l}$, with milky phase creaming on the top of the clear solution. In this case phase separation occurs at concentration above cmc.

Comparison of spreading factors for superspreader BT-278 and catanionic mixtures is given in Fig. 6. The spreading factor decreases with the decrease of the chain length from SOcS to SHexS due to increase of water/air surface tension: SDoS is C12, SDeS is C10, SOcS is C8, SHepS is C7 and SHexS is C6. 


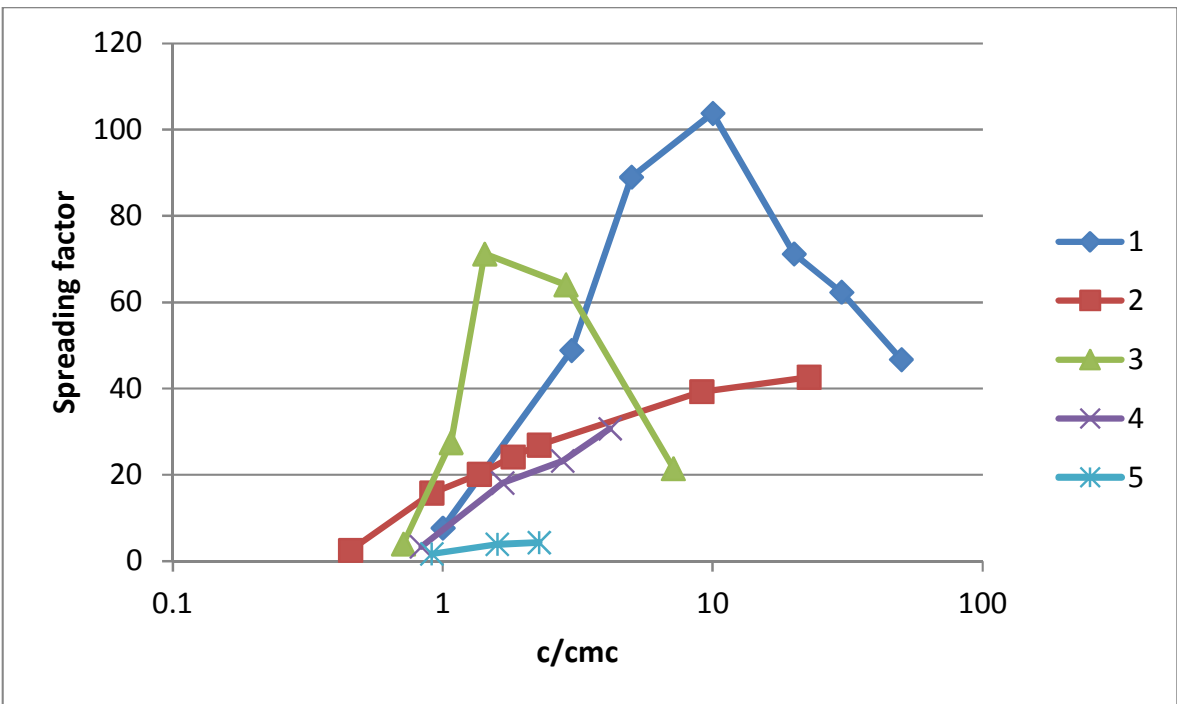

Fig. 6.Spreading factors calculated for $14 \mu$ droplet of $1-B T-278$ and $v: v=1: 1$ mixture of DoTAB with:, 2 - SDeS, 3 - SOcS, 4 - SHepS, 5 - SHexS.

In experiments performed at ambient humidity the spread area reached a maximum at certain concentration of solution and then decreased. It is interesting that the maximum of the spread area for BT-278 and the mixture of DoTAB with SOcSare observed at the identical concentration $1 \mathrm{~g} / \mathrm{l}$. The maximum in the spread area of trisiloxane surfactant at concentration $1 \mathrm{~g} / \mathrm{l}$ agrees with the results presented in [23] for Silvet L-77, which is similar to BT-278.

Note, at concentrations above those presented in Fig. 6 there is a transition from complete to partial wetting even for the superspreader. For example, the solution of BT-278 with concentration $100 \mathrm{~g} / \mathrm{l}$ (which is above both cac/cwc $\sim 1000 \mathrm{cac}$ or 400 cwc) had the contact angle on PE around $20^{\circ}$. After some time, this sessile droplet of this solution shrunk due to evaporation leaving an area covered by trisiloxane only; surprisingly this remaining area is not wetted by more diluted solutions of BT-278: droplet of solution of $1 \mathrm{~g} / \mathrm{l}$ (which is $10 \mathrm{cac}$ or $4 \mathrm{cwc}$ ), which demonstrated the best wetting properties on polyethylene, spread around this area and avoided the deposit, which remained after evaporation of $100 \mathrm{~g} / \mathrm{l}$ solution. That means the deposit was much more hydrophobic tham polyethylene itself. 
The picture changes considerably at higher humidity. In this case the spread area increased with concentration in the whole studied concentration range. At $\mathrm{RH}=40 \%$ the spread area of BT-278 at concentration $10 \mathrm{~g} / \mathrm{l}$ (100 cac) was about 5 times smaller than that at concentration $1 \mathrm{~g} / \mathrm{l}(10 \mathrm{cac})$. At $\mathrm{RH}=80 \%$ spread area increased for both concentration, but only doubled for $1 \mathrm{~g} / \mathrm{l}$ (10 cac), whereas more than 10 times increase was detected for $10 \mathrm{~g} / \mathrm{l}$ (100 cac), i.e. at this high humidity spread area of $10 \mathrm{~g} / \mathrm{l}$ (100 cac) solution was about 1.5 times larger than that of $1 \mathrm{~g} / \mathrm{l}$ (10 cac) solution. Considerably larger increase in the spread area for more concentrated solutions shows that the increase in the spread area caused by the increase of humidity was the result not only of the slower evaporation, but it was related to the change in the spreading properties of solution itself: the thickness of spread film in the end of the spreading process of $10 \mathrm{~g} / \mathrm{l}$ (100 cac) solution of BT-278 was about 20 $\mu \mathrm{m}$ at $\mathrm{RH}=40 \%$ and it decreased to $1-2 \mu \mathrm{m}$ at $\mathrm{RH}=80 \%$. Spreading time was of order of $10 \mathrm{~s}$ for both concentrations at $\mathrm{RH}=40 \%$, after that time the spread area began to decrease because of evaporation for $1 \mathrm{~g} / \mathrm{l}(10 \mathrm{cac})$ solution. For concentration $10 \mathrm{~g} / \mathrm{l}$ (100 cac) evaporation occurred much slower because of much smaller radius of the spread film: liquid evaporated completely within 10-15 min. At $\mathrm{RH}=80 \%$ spreading time of $1 \mathrm{~g} / \mathrm{l}(10 \mathrm{cac})$ solution remained the same around $10 \mathrm{~s}$, but $10 \mathrm{~g} / \mathrm{l}(100 \mathrm{cac})$ solution spread over $10 \mathrm{~min}$. It is interesting that if a substrate with a spread at $\mathrm{RH}=40 \%$ droplet of $\mathrm{BT}-278$ at concentration of $10 \mathrm{~g} / \mathrm{l}$ (100 cac) was moved to the chamber with higher humidity $(80 \%)$ it started to spread further with spread area increasing 8-10 times during around $10 \mathrm{~min}$.

For the mixture SOcS with DoTAB the total spreading time was about 2 min independently of humidity. In this case it looks like the spreading stopped, because dewetting came into play. Nevertheless, similarly to BT-278 the spread area increased with the increase of humidity and the increase was more pronounced at higher concentrations: spread area increased by about $50 \%$ for concentration $1 \mathrm{~g} / \mathrm{l}$ $(1.4 \mathrm{cmc})$ and 4-5 times for concentration $10 \mathrm{~g} / \mathrm{l}(14 \mathrm{cmc})$ at the increase of humidity from $40 \%$ to $80 \%$. The scattering in the results was very high in this case and the difference in the spread area for $1 \mathrm{~g} / \mathrm{l}(1.4 \mathrm{cmc})$ and $10 \mathrm{~g} / \mathrm{l}(14 \mathrm{cmc})$ at $\mathrm{RH}=80 \%$ is in the range of experimental error with average being a little bit higher for $10 \mathrm{~g} / \mathrm{l}$ (14 $\mathrm{cmc}$ ). 
During spreading of BT-278 a circular shape retained all the time with the small fingers appearing before the end of the spreading. The shape of spread of catanionic mixture solution was less regular. After the maximum area had been reached, the dewetting was observed for all catanionic solutions: the spread film retreated reassembling back in the droplet. The reassembling process was more pronounced at higher concentrations. This phenomenon was already described earlier for the mixture of SDeS with DoTab [20]. In that case the reassembled drop had a large contact angle (about $50^{\circ}$ ) and the phenomenon was related to the increase of surface tension due to crystallisation. The crystallization did not occur in mixed solutions of DoTAB with sodium alkane sulphonates with hydrophobic chain length 8 and less than 8 hydrocarbon groups. Liquid/liquid phase separation was observed for SOcS at concentrations $5 \mathrm{~g} / \mathrm{l}(67 \mathrm{cmc})$ and above. Mixed solutions with SHepS remained clear till concentration $10 \mathrm{~g} / \mathrm{l}(6 \mathrm{cmc})$. Nevertheless the dewetting was observedeven in this case. Note, in two last cases contact angle remained very small after dewetting.

Short time spreading kinetics of solution BT-278 is presented in Fig. 7 and that of the most effective catanionic mixture SOcS+DoTAB in Fig. 8. Note, the inertial or viscous stages of spreading, which develop on shorter time scale [24,25] were not considered: only kinetics of spreading on the time scale from hundred milliseconds to seconds was investigated, i.e. during the superspreading stage [24]. Experimental errors at measurements of spreading kinetics at the early stage were smaller for BT278 (not exceed $\pm 10 \%$ ) than for catanionic mixture ( $\pm 20 \%$ ). Kinetics virtually did not change at the change in humidity: difference in kinetics between $\mathrm{RH}=40 \%$ and $\mathrm{RH}=80 \%$ was in the range of experimental error.

Figs. 7 and 8 show that at concentrations below $10 \mathrm{~g} / \mathrm{l}$ (100 cac) for BT-278 and below $5 \mathrm{~g} / \mathrm{l}(6.7 \mathrm{cmc})$ for the mixture SOcS+DoTAB spread area increased linearly with time over the studied time interval, but at larger concentrations spreading slowed down after the linear part. The same slowing down was found for other concentrations as well, but occurred at larger times as it is presented in Fig. 9, where the long-time kinetics is presented for some selected solutions. For BT-278, $10 \mathrm{~g} / \mathrm{l}$ (100 cac) the linear stage is absent in Fig. 10, because it ended much earlier as it is shown in Fig. 7. This slowing down is the reason why spread area demonstrates a maximum vs concentration at $40 \%$ humidity. 


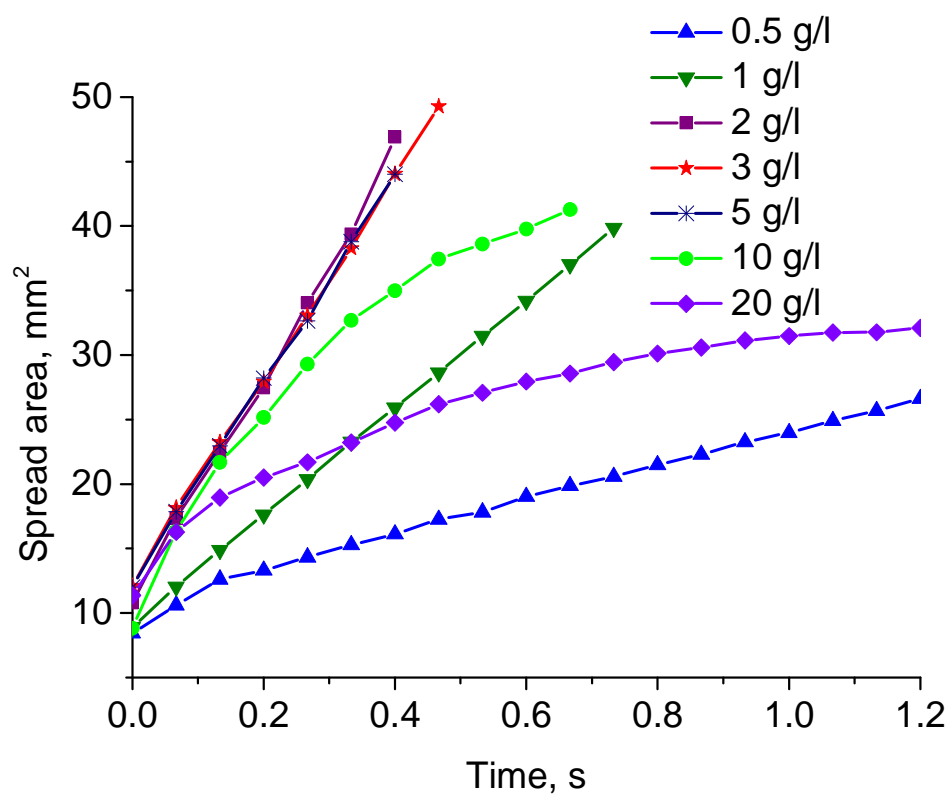

Fig. 7. Kinetics of spreading of BT-278 trisiloxane superspreader at various concentrations. Droplet volume $2 \mu$.

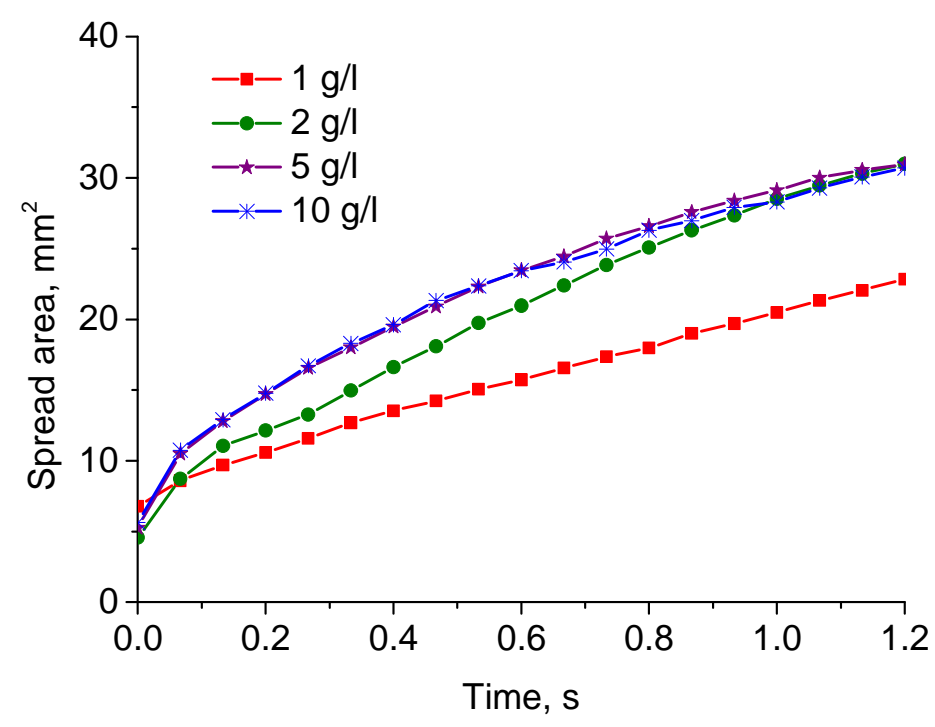


Fig. 8. Kinetics of spreading of mixture SOcS+DoTAB at various concentrations. Droplet volume $2 \mu \mathrm{l}$.

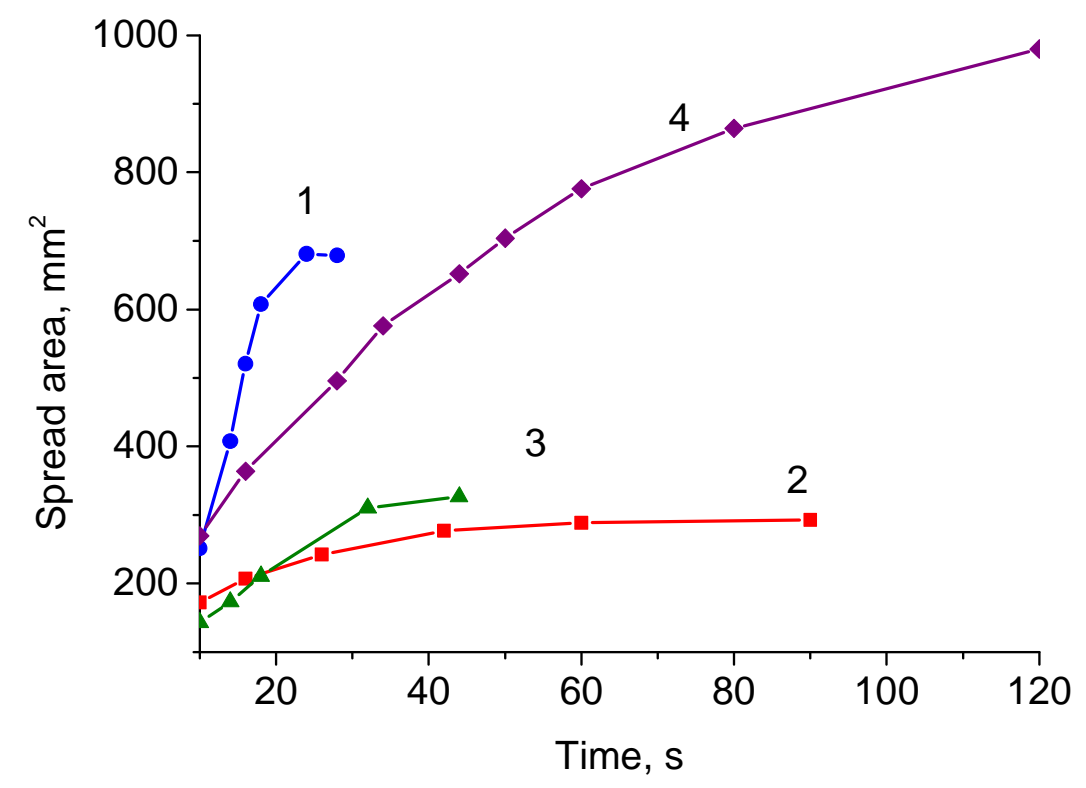

Fig. 9. Long time kinetics of spreading: 1 - BT-278, $0.3 \mathrm{~g} / \mathrm{l} ; 2-\mathrm{BT}-278,10 \mathrm{~g} / \mathrm{l} ; 3-$ SHepS+ DoTAB, $10 \mathrm{~g} / \mathrm{l}, 4$ - SOcS+DoTAB $1 \mathrm{~g} / \mathrm{l}$. Droplet volume $14 \mu \mathrm{l}$.

Concentration dependences of the short time spreading rate for BT-278 and mixture SOcS+DoTAB are summarised in Fig. 10. The values shown in Fig. 10 are the averaged values during the first recorded second of spreading. The essential decrease in the spreading rate of BT-278 at concentrations 10 and $20 \mathrm{~g} / \mathrm{l}$ are the result of the early slowing down of the spreading. If we consider the spreading rate as an initial slope of curves in Figs. 8 and 9 then it can be concluded that it increases and reaches plateau at concentrations above $2 \mathrm{~g} / \mathrm{l}$ for both surfactants (see Figs. 7 and 8).

Note, the spreading rate for the mixture SOcS+DoTAB is nearly 5 times lower than that for BT-278 despite the same spreading exponent $\alpha=0.5$. That is, factor $A_{1}$ in Eq. (1a) is much larger for solutions BT-278 as compared with catanionic mixtures. The mechanism of spreading and the big large difference in factor $A_{1}$ for different 
surfactants is to be explained. Note, the case of the spreading of pure liquids $A_{1}$ does not vary considerably [6]. For example, the difference in $A_{1}$ at spreading of silicone oil over PE (contact angle of water 1019 ) and clean glass(contact angle of water close to 0 ) is only about $12 \%$ despite the large difference in the energy of substrates.

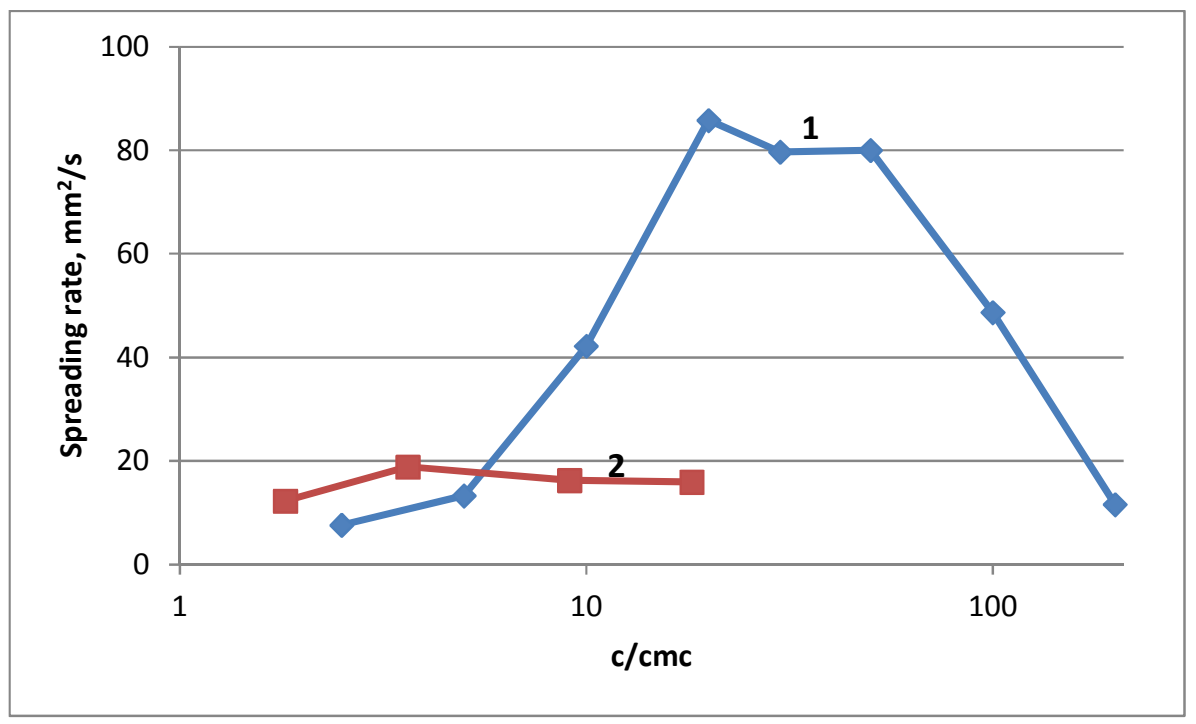

Fig. 10.Short time spreading rate vs concentration for $1-\mathrm{BT}-278$ and $2-$ mixture of DoTAB+SOcS solutions.

\section{Conclusions}

Mixtures of dodecyltrimethylammonium bromide with sodium alcane sulfonates C6C10 demonstrated essential synergism in surface activities and in wetting performance. The best wetting inside $v: v=1: 1$ mixtures was achieved with DoTAB+SOcS at concentration $1 \mathrm{~g} / \mathrm{l}(\sim 1.4 \mathrm{cmc})$. Comparative study performed at the same conditions has shown that spreading factor of this mixture reaches about $70 \%$ of spreading factor of trisiloxane superspreader BREAK-THRU S 278.

Spreading rate of catanionic mixtures is considerably lower as compared with BT278: the maximum spreading rate of DoTAB+SOcS mixture is nearly 5 times smaller 
than that of BT-278. At the same time spreading of mixtures lasts longer, resulting in comparable with BT-278 spread areas.

Initial rate of spreading increased with surfactant concentration until the maximum was reached for all surfactants solutions and in the range of concentrations studied, and then initial rate of spreading started to decrease slowly. At the same time the spread area went via maximum value. The spread area is determined by both spreading rate and the time of spreading. It was found that the spreading process started to slow down earlier at higher concentrations.

An increase in humidity resulted in an increase of spreading time and consequently in the increase of the spread area. The increase in the spread area caused by the increase of humidity was not only result of slower evaporation rate, but it was also related to the change in the spreading properties of the solution itself.

\section{Acknowledgments}

Superspreader BT-278 was donated by Evonik Industries for this research. Special thanks to Dr $\mathrm{J}$ Venzmer for supplying the surfactants. This research was supported by Engineering and Physical Sciences Research Council, UK, grant EP/D077869/1; by CoWet Marie Curie, EU project; by European Space Agency, MAP, FASES and PASTA projects; and by COST MP1106 EU project.

\section{References}

1. Hill R.M. Superspreading. COCIS, 1998, 3, 247-254.

2. Venzmer J. Superspreading - 20 years of physic-chemical research. COCIS, $2011,16,335-343$.

3. Svitova T., Hill R.M., Smirnova Yu., Stuermer A., Yakubov G. Wetting and interfacial transitions in dilute solutions of trisiloxane surfactants. Langmuir, 1998, 14, 5023-5031.

4. Kumar N., Couzis A., Maldarelli C. Measurement of kinetic rate constants for the adsorption of superspreadingtrisiloxanes to an air/aqueous interface and the relevance of these measurements to the mechanism of superspreading. J. Coll. Int. Sci., 2003, 267, 272-285. 
5. Ritacco H.A., Ortega F., Rubio R.G., Ivanova N., Starov V.M. Equilibrium and dynamic surface properties of trisiloxane aqueous solutions. Part. 1. Experimental results. Coll. Surf. A, 2010, 365, 199-203.

6. Starov V.M., Kalinin V.V., Chen J.D. Spreading of liquid drops over dry surfaces. Adv. Coll. Int. Sci. 1994, 50, 187-221.

7. Tanner L.H. The spreading of silicone oil droplets on horizontal surfaces. J. Phys. D: Appl. Phys. 1979, 12, 1473-1484.

8. Kovalchuk N.M., Trybala A., Starov V., Matar O, IvanovaN.Fluoro- vs hydrocarbon surfactants: Why do they differ in wetting performance? Adv. Coll. Int. Sci. 2014, 210, 65-71.

9. Tiberg F., Cazabat A.-M. Self-assembly and spreading of non-ionic trisiloxane surfactants. Europhys.Lett. 1994, 25, 205-210.

10. P. G. de Gennes. Wetting: statics and dynamics. Rev. Mod. Phys., 1985, 57, 827.

11. V.Starov. Static contact angle hysteresis on smooth, homogeneous solid substrates. Colloid Polym Sci, 2013, 291, 261-270.

12. G.A. Martynov, V.M. Starov, N.V. Churaev. Contact Angle Hysteresis at Homogeneous Surfaces. Colloid J. of the USSR, (English Translation), 1977, 39, 406-417.

13. Nikolov A.D., Wasan D., Chengara A., Koczo K., Pociello G.A., Kolossvary I. Superspreading driven by Marangoni flow. Adv. Coll. Int. Sci., 2002, 96, 325-338. 14. Lin Z., Hill R.M., Davis H.T., Ward M.D. Determination of wetting velocities of surfactant superspreaders with the quartz crystal microbalance. Langmuir, 1994, 10, 4060-4068.

15. Zhu S., Miller W.G., Scriven L.E., Davis H.T. Superspreading of water-silicone surfactant on hydrophobic surfaces. Coll. Surf. A, 1994, 90, 63-78. 
16. Stoebe T., Lin Z., Hill R.M. Ward M.D., Davis H.T. Enhanced spreading of aqueous films containing ethoxilated alcohol surfactants on solid substrates. Langmuir, 1997, 13, 7270-7275.

17. Stoebe T., Hill R.M. Ward M.D., Davis H.T. Enhanced spreading of aqueous films containing ionic surfactants on solid substrates. Langmuir, 1997, 13, 7276-8281.

18. Rosen M.J. Predicting synergism in binary mixtures of surfactants. Progr. Coll. Polymer Sci. 1994, 95, 39-47.

19. Wu Y., Rosen M.J. Synergism in the spreading of hydrocarbon -chain surfactants on polyethylene film - anionic and cationic mixtures by a two-step procedure. Langmuir, 2005, 21, 2342-2348.

20. Kovalchuk N.M., Barton A., Trybala A., Starov V. Surfactant enhanced spreading: catanionic mixture. Coll. Int. Sci. Comm. 2014, 1, 1-5.

21. Kwok D.Y., Neumann A.W. Contact angle measurement and contact angle interpretation. Adv. Coll. Int. Sci., 1999; 81: 167-249.

22. Pradines V., Fainerman V.B., Aksenenko E.V., Kragel J., Mucic N., Miller R. Adsorption of alkyl trimethylammonium bromides at water/air and water hexane interfaces. Coll. Sur. A 2010, 371, 22-28.

23. Chengara A., Nikolov A., Wasan D. Surface tension driven spreading of trisiloxane surfactant solution on hydrophobic solid. Coll. Surf. A, 2002, 206, 31-39.

24. Wang. X., Chen L., Bonaccurso E., Venzmer J. Dynamic wetting of hydrophobic polymers by aqueous surfactant and superspreader solutions. Langmuir, 2013, 29, 14855-14864.

25. Chen L., Bonaccurso E. Effect of surface wettability and liquid viscosity on the dynamic wetting of individual drops. Phys. Rev. E, 2014, 90, 022401. 


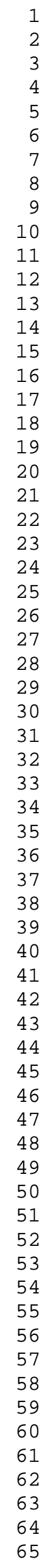

